\title{
CHANDRA, KECK, AND VLA OBSERVATIONS OF THE CRAB NEBULA DURING THE 2011-APRIL GAMMA-RAY FLARE
}

\author{
Martin C. Weisskopf ${ }^{1}$, Allyn F. Tennant ${ }^{1}$, Jonathan Arons ${ }^{2}$, Roger Blandford ${ }^{3}$, Rolf Buehler ${ }^{4}$, \\ Patrizia Caraveo $^{5,6}$, Chi C. Cheung ${ }^{7,17}$, Enrico Costa ${ }^{8}$, Andrea de Luca ${ }^{5,6}$, Carlo Ferrigno $^{9}$, Hai Fu $^{10}$, \\ Stefan FunK ${ }^{3}$, Moritz Habermehl ${ }^{11}$, Dieter Horns ${ }^{11}$, Justin D. Linford ${ }^{12}$, Andrei Lobanov ${ }^{13}$, Claire MaX ${ }^{14}$, \\ Roberto Mignani ${ }^{15}, 16$, Stephen L. O’Dell ${ }^{1}$, Roger W. Romani ${ }^{3}$, Edoardo Striani ${ }^{8}$, Marco Tavani ${ }^{8}$, \\ GREgORY B. TAYLOR ${ }^{12}$, YASUNOBU UChIYAMa ${ }^{3}$, AND YAJIE YUAN ${ }^{3}$ \\ ${ }^{1}$ NASA Marshall Space Flight Center, Astrophysics Office (ZP12), Huntsville, AL 35812, USA \\ ${ }^{2}$ Astronomy Department and Theoretical Astrophysics Center, University of California, Berkeley, 601 Campbell Hall, Berkeley, CA 94720, USA \\ ${ }^{3}$ W. W. Hansen Experimental Physics Laboratory, Kavli Institute for Particle Astrophysics and Cosmology, \\ Department of Physics and SLAC National Accelerator Laboratory, Stanford University, Stanford, CA 94305, USA \\ ${ }^{4}$ DESY, Platanenallee 6, D-15738 Zeuthen, Germany \\ ${ }^{5}$ INAF-IASF Milano, via E. Bassini 15, I-20133 Milano, Italy \\ ${ }^{6}$ INFN Pavia, via A. Bassi 6, I-27100 Pavia, Italy \\ ${ }^{7}$ National Research Council Research Associate, National Academy of Sciences, Washington, DC 20001, USA \\ ${ }^{8}$ INFN Roma Tor Vergata, via della Ricerca Scientifica 1, I-00133 Roma, Italy \\ Received 2012 November 9; accepted 2012 December 27; published 2013 February 15
}

\begin{abstract}
We present results from our analysis of Chandra X-Ray Observatory, W. M. Keck Observatory, and Karl G. Jansky Very Large Array (VLA) images of the Crab Nebula that were contemporaneous with the $\gamma$-ray flare of 2011 April. Despite hints in the X-ray data, we find no evidence for statistically significant variations that pinpoint the specific location of the flares within the Nebula. The Keck observations extend this conclusion to the "inner knot," i.e., the feature within an arcsecond of the pulsar. The VLA observations support this conclusion. We also discuss theoretical implications of the $\gamma$-ray flares and suggest that the most dramatic $\gamma$-ray flares are due to radiation-reaction-limited synchrotron emission associated with sudden, dissipative changes in the current system sustained by the central pulsar.
\end{abstract}

Key words: gamma rays: ISM - infrared: ISM - ISM: individual objects (Crab Nebula, M1) - magnetic reconnection - radio continuum: ISM - X-rays: individual (Crab Nebula, M1)

\section{INTRODUCTION}

The Crab Nebula, the relic of a stellar explosion recorded by Chinese astronomers in 1054, has a special place in the history of astronomy. It is our most frequently observed laboratory for high-energy astrophysics. Located at a distance of $\approx 2 \mathrm{kpc}$, the system is energized by a pulsar of spin-down luminosity $L_{\mathrm{plsr}} \approx 5 \times 10^{38} \mathrm{erg} \mathrm{s}^{-1}$ and current spin period $P \approx 34 \mathrm{~ms}$. The history and general properties of the system are nicely summarized in the review by Hester (2008). Optical and X-ray images (Hester et al. 1995, 2002; Weisskopf et al. 2000) of the inner nebula show features such as an inner ring, toroidal structure, knots, and two opposing jets originating from the pulsar - these latter presumably aligned with its rotation axis and proper motion vector (Caraveo \& Mignani 1999; Ng \& Romani 2007; Kaplan et al. 2008 and references therein). The "inner-ring," prominent in X-rays, is commonly accepted as being the termination shock produced by the relativistic wind of particles accelerated by the pulsar. Many of the optical and X-ray features brighten and fade and/or move over weeks or months (e.g., Hester et al. 1995, 2002).

\footnotetext{
${ }^{17}$ Resident at Naval Research Laboratory, Washington, DC 20375, USA.
}

The quiescent or average spectral energy distribution (SED) of the Crab Nebula has a characteristic two-humped form (see, e.g., Figure 8; Atoyan \& Aharonian 1996; Bucciantini et al. 2011 and references therein). The synchrotron spectrum extends from $\approx 30 \mathrm{MHz}$ to $\approx 1.2 \times 10^{22} \mathrm{~Hz}(500 \mathrm{MeV})$. Most of the power is radiated by $\approx \mathrm{TeV}$ electrons in the near $\mathrm{UV} \approx 10 \mathrm{eV}$ with an associated luminosity of $\approx 1.3 \times 10^{38} \mathrm{erg} \mathrm{s}^{-1}$ (Hester 2008). This roughly matches the loss of rotational energy by the pulsar, which releases its energy electromagnetically, generating a current $\approx 200 \mathrm{TA}$ and inducing an electro-motive force (EMF) $\approx 50 \mathrm{PV}$. However, the nebula is currently varying on a fewyear timescale (Wilson-Hodge et al. 2011). At higher energies, Compton scattering has a luminosity of $\approx 10^{36} \mathrm{erg} \mathrm{s}^{-1}$, peaking around $60 \mathrm{GeV}$ (Albert et al. 2008) and measured up to $\approx 80 \mathrm{TeV}$ (e.g., Aharonian et al. 2004; Abdo et al. 2010).

Since 2007, the AGILE and Fermi satellites have detected several $\gamma$-ray flares from the Crab Nebula (Tavani et al. 2011; Abdo et al. 2011; Striani et al. 2011a; Buehler et al. 2012) in the $0.1-1 \mathrm{GeV}$ range. The most dramatic flares exhibit variability on timescales as short as a few hours, although it is unclear whether they are distinct events or just the largest variations from a stationary power spectrum of fluctuations. Prior to the 2011-April event, the only Crab $\gamma$-ray flare covered by 
Table 1

Time-ordered List of Chandra Observations and Fermi-LAT $\gamma$-Ray Measurements ${ }^{\mathrm{a}}$

\begin{tabular}{ccccc}
\hline \hline ObsID & Date $^{\mathrm{b}}$ & $\Gamma_{\gamma}{ }^{\mathrm{c}}$ & $N(>100 \mathrm{MeV})^{\mathrm{d}}$ & $N_{E}(100 \mathrm{MeV})^{\mathrm{e}}$ \\
\hline 13150 & 4851.039 & $2.42 \pm 0.08$ & $(1.27 \pm 0.08) \times 10^{-5}$ & $(1.80 \pm 0.15) \times 10^{-10}$ \\
13151 & 4851.667 & $2.25 \pm 0.15$ & $(8.05 \pm 0.15) \times 10^{-6}$ & $(1.01 \pm 0.12) \times 10^{-10}$ \\
13152 & 4853.423 & $2.20 \pm 0.06$ & $(1.54 \pm 0.06) \times 10^{-5}$ & $(1.84 \pm 0.12) \times 10^{-10}$ \\
13153 & 4859.032 & $2.27 \pm 0.21$ & $(4.68 \pm 0.21) \times 10^{-6}$ & $(6.0 \pm 1.0) \times 10^{-11}$ \\
13154 & 4865.335 & $2.76 \pm 0.40$ & $(4.50 \pm 0.40) \times 10^{-6}$ & $(7.9 \pm 1.9) \times 10^{-11}$
\end{tabular}

Notes.

a Analyzed following all the procedures in Buehler et al. (2012).

${ }^{\mathrm{b}}$ Days after MJD 50814 to the middle of the observation, which is $10 \mathrm{ks}$ long.

${ }^{c} \gamma$-ray power law number index. The error is the maximum of the two-sided uncertainty.

${ }^{\mathrm{d}}$ Photon integrated flux $\left[\mathrm{ph}\left(\mathrm{cm}^{2} \mathrm{~s}\right)^{-1}\right]$ above $100 \mathrm{MeV}$. The error is the maximum of the two-sided uncertainty.

e Photon spectral flux $\left[\mathrm{ph}\left(\mathrm{cm}^{2} \mathrm{~s} \mathrm{keV}\right)^{-1}\right]$ at $100 \mathrm{MeV}$.

a multi-wavelength observing program was the 2010-September flare, which triggered observations in radio, optical (using both ground-based telescopes and Hubble Space Telescope; HST), and X-ray bands. Despite the $\gamma$-ray brightness of the flares, there has been no evidence for correlated variations in radio (Lobanov et al. 2011; this paper), near infrared (Kanbach et al. 2010; this paper), optical (Caraveo et al. 2010), or X-ray bands (Evangelista et al. 2010; Shaposhnikov et al. 2010; Tennant et al. 2010, 2011; Ferrigno et al. 2010; Horns et al. 2010; Cusumano et al. 2011; Tavani et al. 2011; Striani et al. 2011b; this paper).

Here we focus on the Fermi-LAT results for the 2011-April flare (Buehler et al. 2012), which allow us to assess the source behavior in detail. The source doubled its $\gamma$-ray flux within eight hours and reached a peak flux 30 times its average. The isotropic luminosity increased to $\approx 2 \times 10^{37} \mathrm{erg} \mathrm{s}^{-1}$ in $\approx 10 \mathrm{hr}$ and the spectrum peaked at $\approx 400 \mathrm{MeV}$. Table 1 gives the $\gamma$-ray power law photon spectral index, the integrated photon flux above $100 \mathrm{MeV}$, and the photon spectral flux at $100 \mathrm{MeV}$, as measured during the $10 \mathrm{ks}$ time intervals when X-ray data (Section 2) were taken.

Notification as to the level of flaring prompted us to trigger pre-approved Target of Opportunity observations with Chandra and with the NRAO ${ }^{18}$ Karl G. Jansky Very Large Array (VLA). We were also fortunate to obtain a Keck image in the near infrared, albeit not under ideal conditions. Figure 1 shows the Fermi-LAT $\gamma$-ray counting rate as a function of time and also indicates the times of the Chandra, Keck (Section 3), and some of the VLA (Section 4) observations.

\section{X-RAY OBSERVATIONS AND DATA ANALYSIS}

With the back-illuminated ACIS S3 CCD on the Chandra $X$-Ray Observatory approximately centered on the Crab pulsar, we obtained five observations (Table 1) during and somewhat after the 2011 April $\gamma$-ray flare. For these observations, the spacecraft dithered with an amplitude set to $1^{\prime \prime}$. Although standard processing typically produces an aspect solution better than 0.5 , even this small uncertainty can introduce noticeable shifts when comparing different data sets. Thus, we re-registered images for our analysis using the read-out streak and the pulsar as guides. As each of these five images was placed at approximately the same CCD location, spatial non-uniformity in the ACIS response (e.g., due to contamination) does not introduce spurious temporal variability.

\footnotetext{
18 The National Radio Astronomy Observatory is a facility of the National Science Foundation operated under cooperative agreement by Associated Universities, Inc.
}

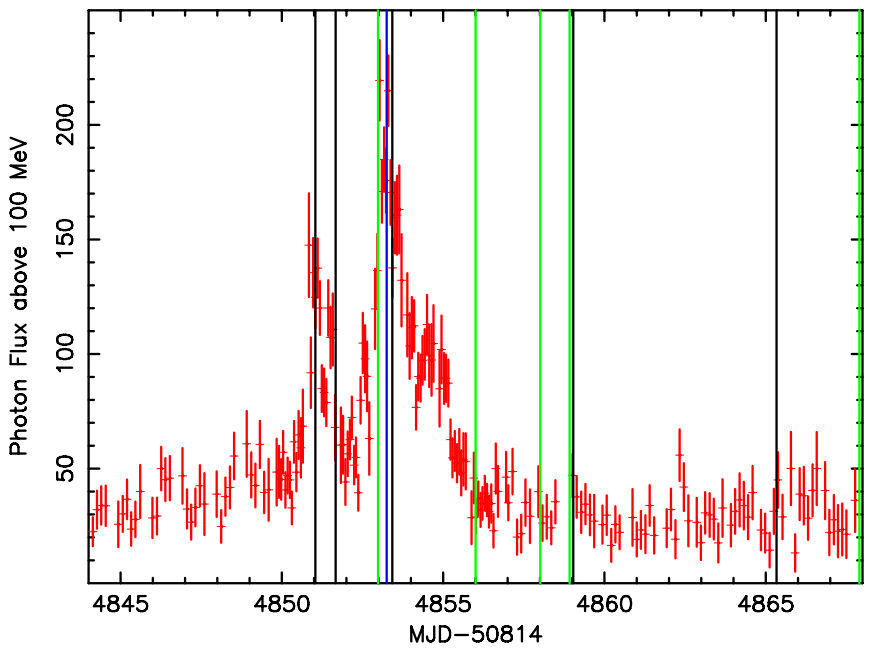

Figure 1. Fermi-LAT photon flux $\left(10^{-7} \mathrm{ph}\left(\mathrm{cm}^{2} \mathrm{~s}\right)^{-1}\right)$ above $100 \mathrm{MeV}$ during the 2011 April flare as a function of time. Displayed data extend beyond the time span shown in Buehler et al. (2012) but follow the same data processing as described there: Data are adaptively binned with a 20 minute average bin duration. The full-range vertical lines denote times of the five $(\approx 1200 \mathrm{~s})$ Chandra observations (black), of the Keck observation (blue, fourth from left), and of the first five VLA observations (green).

Owing to the Crab's high flux, the ACIS observations employed a special mode with $0.2 \mathrm{~s}$ frame time, which limits the CCD read-out to a $300 \times 300$ ACIS-pixel $\left(\approx 150^{\prime \prime} \times 150^{\prime \prime}\right)$ subarray. Although each observation lasted about $10 \mathrm{ks}$, telemetry saturation reduced the effective integration time to approximately $1200 \mathrm{~s}$ per observation. Despite the short frame time of the special ACIS mode, regions of high surface brightness suffer somewhat from pile-up effects. We consider only data in the range $0.5-8.0 \mathrm{keV}$ because of severe interstellar absorption at low energies and declining flux at high energies. Using these data, we then search for X-ray variations approximately contemporaneous with the 2011-April $\gamma$-ray flare.

\subsection{X-Ray Image Analysis}

Figure 2 shows an image of the number of counts per ACIS pixel, summed over the five observations. For each observation, we re-binned a $120 \times 120$ ACIS-pixel image centered on the pulsar into a $60 \times 60$ array of $2 \times 2$ ACIS pixels. Each of these $I=$ 3600 "analysis pixels" is sufficiently large (about $1 \operatorname{arcsec}^{2}$ ) to enclose most of the Chandra point-spread function anywhere in the field of view. Note that we also performed an analysis using a circular bin of radius 1 ACIS pixel on an oversampled grid of 


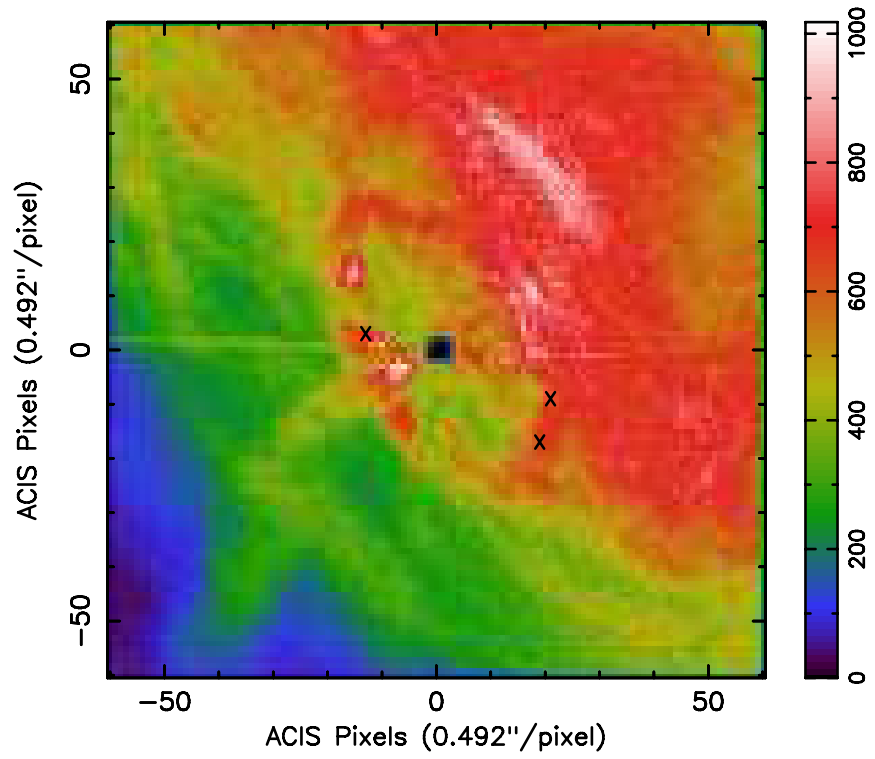

Figure 2. Summed image for the five Chandra ACIS observations occurring near the 2011 April $\gamma$-ray flare, at the native CCD resolution. The color bar gives summed counts per ACIS pixel over a total effective exposure of about $6 \mathrm{ks}$. North is up and the pulsar is at $(0,0)$ in the displayed ACIS-pixel coordinates. The nearly horizontal read-out streak through the pulsar's location is the trailed (out-of-time) image, resulting from exposure of each CCD pixel as the image is read out at $40 \mu$ s per row. As the five observations occurred at slightly different roll angles, the read-out streak is slightly blurred azimuthally. The X symbols mark locations of the three statistically most significant variations $\left(S_{i}>6\right.$; Section 2.2), the most significant lying to the east of the pulsar.

cadence $0.1 \times$ ACIS pixel-i.e., a spherical top hat smoothing of the events. As each method gave similar results, we here report the results for the "analysis pixel" binning, for which each pixel is statistically independent.

For each analysis pixel $i$, we calculate the mean count rate $r_{i}$ averaged over the $J=5$ observations, weighted ${ }^{19}$ by the respective (counting-rate) statistical error $\sigma_{i j}$ for each analysis

${ }^{19} r_{i}=\sum_{j=1}^{J}\left\{r_{i j} / \sigma_{i j}^{2}\right\} / \sum_{j=1}^{J}\left\{1 / \sigma_{i j}^{2}\right\}$.

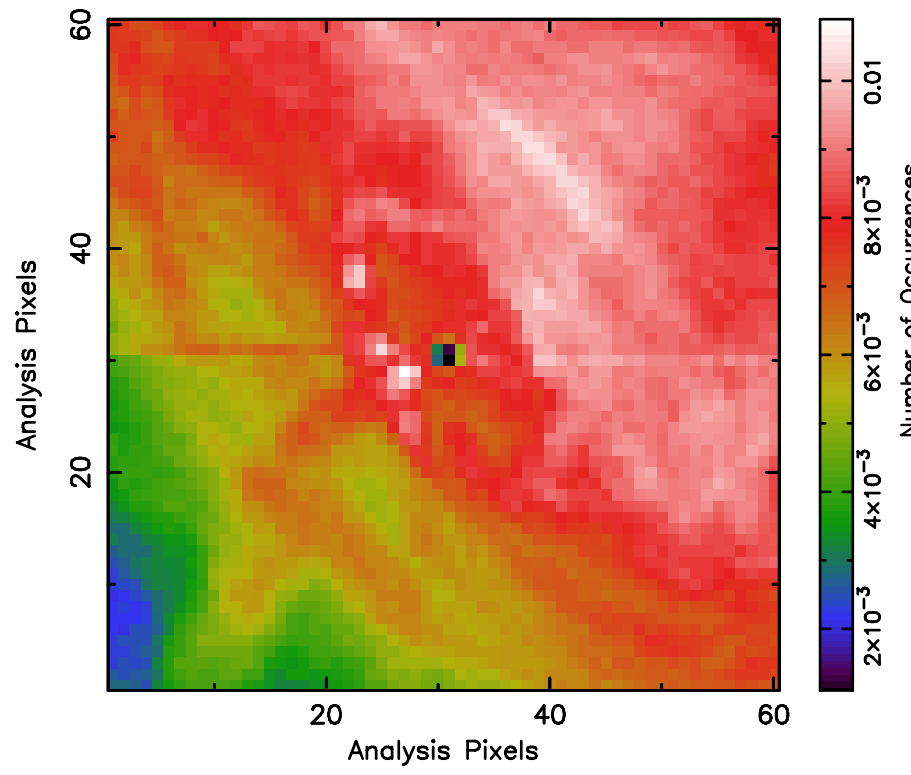

pixel and observation. For evaluating statistical significance of temporal variations over the $J=5$ observations, we compute ${ }^{20}$ $\chi_{i}^{2}$ along with a derived significance measure $S_{i}$.

For purposes of discussion, we also compute the appropriately weighted $^{21}$ statistical error $\sigma_{i}$ and sample standard deviation $s_{i}$ for each pixel $i$. As properly weighted, $\chi_{i}^{2}=(J-1) s_{i}^{2} / \sigma_{i}^{2}$. While we have rigorously calculated $r_{i}, \chi_{i}^{2}, \sigma_{i}$, and $s_{i}$ for each pixel $i$ using appropriate weightings, we note that the weightings are nearly uniform as the effective duration of the each of the $J=5$ observations was nearly the same-about $1200 \mathrm{~s}$.

\subsection{Variability of the X-Ray Images}

The (counting) statistical error $\sigma_{i}$ is the primary noise term and thus governs the sensitivity for detecting temporal variations at the analysis-pixel (square-arcsec) scale. Figure 3 shows the image and the corresponding histogram of the distribution of $\sigma_{i}$, which ranges from 0.0025 to $0.024 \mathrm{ct} \mathrm{s}^{-1}$ per analysis pixel. Based upon the $\chi^{2}$ probability distribution and the number of "tries" ( $I=3600$ independent analysis pixels), a $99 \%$ confidence detection would require a $\chi_{i, 99 \%}^{2}>31.2$ on $(J-1)=4$ degrees of freedom. This corresponds to a sample standard deviation $s_{i, 99 \%}>2.80 \sigma_{i}$, which ranges from 0.0071 to $0.068 \mathrm{ct} \mathrm{s}^{-1}$ over the field.

Here we do not display the analogous image and histogram for the sample standard deviation $s_{i}$, which ranges from 0.0014 to $0.048 \mathrm{ct} \mathrm{s}^{-1}$ per analysis pixel. Instead, Figure 4 shows the image and histogram of the distribution of a calculated (Section 2.1) significance measure $S_{i}$, related to $\chi_{i}^{2}=(J-1) s_{i}^{2} / \sigma_{i}^{2}$. The statistically most significant variation has $\chi_{i}^{2}=23.5$ on $v=(J-1)=4$ degrees of freedom giving $S_{i}=6.9$. Such a fluctuation is expected statistically in at least 1 of 3600 pixels in $31 \%$ of realizations. Table 2 gives the sample standard deviation and $99 \%$-confidence upper limit to the count-rate variation, for the analysis pixel with the statistically most significant X-ray

$$
\begin{array}{ll}
20 & \chi_{i}^{2}=\sum_{j=1}^{J}\left\{\left(r_{i j}-r_{i}\right)^{2} / \sigma_{i j}^{2}\right\} \text { and } S_{i} \equiv\left(\chi_{i}^{2}-v_{i}\right) / \sqrt{2 v_{i}} \text { where } v_{i}=(J-1) . \\
{ }^{21} & \sigma_{i}^{2}=J / \sum_{j=1}^{J}\left\{1 / \sigma_{i j}^{2}\right\} \text { and } \\
s_{i}^{2}=(J /(J-1)) \sum_{j=1}^{J}\left\{\left(r_{i j}-r_{i}\right)^{2} / \sigma_{i j}^{2}\right\} / \sum_{j=1}^{J}\left\{1 / \sigma_{i j}^{2}\right\}=\left(\chi_{i}^{2} /(J-1)\right) \sigma_{i}^{2} .
\end{array}
$$

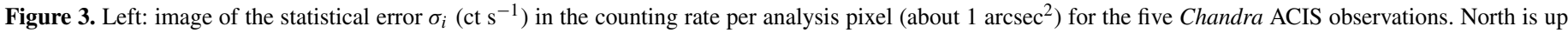
and the pulsar is at $(30,30)$ in the displayed analysis-pixel coordinates. Right: histogram of number of occurrences of each value in the image to the left. 

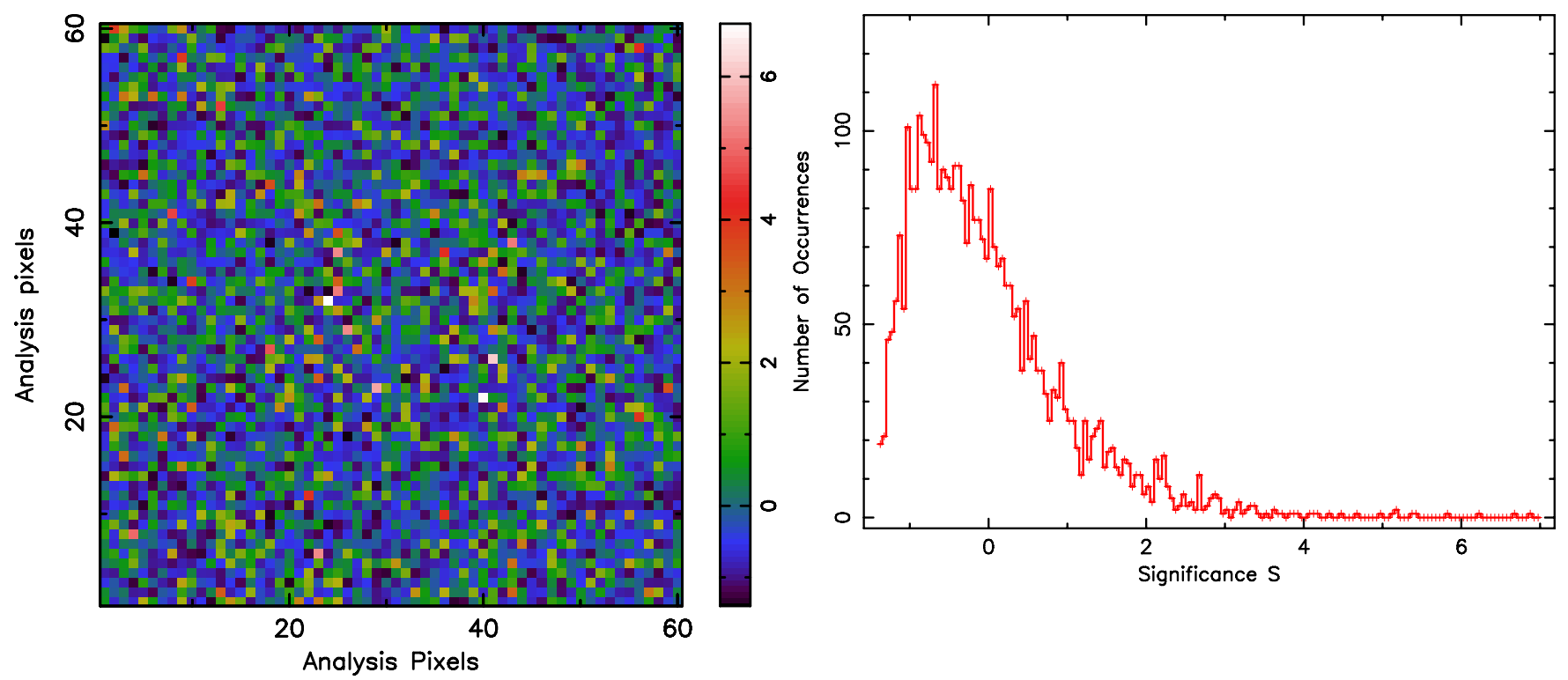

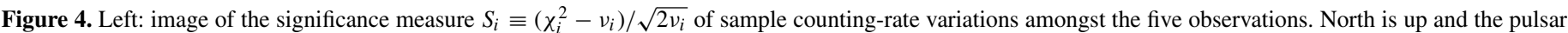
is at $(30,30)$ in the displayed analysis-pixel coordinates. Right: histogram of number of occurrences of each value in the image to the left.

Table 2

X-Ray Results at $1 \mathrm{keV}$ for the Analysis Pixel with the Most Significant Variation

\begin{tabular}{|c|c|c|c|c|c|c|c|}
\hline \multirow{2}{*}{$\begin{array}{l}\text { Quantity } \\
\text { Rate } \\
\Gamma_{x}\end{array}$} & \multirow[t]{2}{*}{$\begin{array}{l}\text { Unit } \\
\text { ct s}^{-1}\end{array}$} & \multicolumn{3}{|c|}{$\begin{array}{c}\text { Sample stdev }(s) \\
0.0480\end{array}$} & \multicolumn{3}{|c|}{$\begin{array}{c}99 \% \text {-Upper Limit } \\
0.0554\end{array}$} \\
\hline & & $\frac{2}{3}$ & 1 & 2 & $\frac{2}{3}$ & 1 & 2 \\
\hline$N_{E}$ & $10^{-4} \mathrm{ph}\left(\mathrm{cm}^{2} \mathrm{~s} \mathrm{keV}\right)^{-1}$ & 0.48 & 0.61 & 1.18 & 0.55 & 0.70 & 1.36 \\
\hline$F_{E}$ & $10^{-13} \operatorname{erg}\left(\mathrm{cm}^{2} \mathrm{~s} \mathrm{keV}\right)^{-1}$ & 0.76 & 0.97 & 1.89 & 0.88 & 1.12 & 2.18 \\
\hline$E L_{E}$ & $10^{32} \mathrm{erg} \mathrm{s}^{-1}$ & 0.37 & 0.47 & 0.91 & 0.42 & 0.54 & 1.05 \\
\hline$\Gamma_{x \gamma}$ & & 1.18 & 1.20 & 1.26 & 1.20 & 1.22 & 1.27 \\
\hline
\end{tabular}

variation. While we detect no variations statistically significant at $99 \%$ confidence, it is curious that the three most significant variations occur at locations on the inner ring.

Note that if a feature, such as one of the knots, does not change in intensity but moves from one analysis pixel to another, then our $\chi_{i}^{2}$ test would detect this as a variation. We know that features in the inner ring of the Nebula do move and expect to detect some variability due to this motion. However, as our five observations span only 14 days and 1 " corresponds to 11.5 light days, only relativistic motion would be detectable. Other effects, such as changes in the roll angle of the read-out streak, can also lead to spurious variability. Indeed, this may play a role for the analysis pixel with the most significant variation, which lies adjacent to the average read-out streak (Figure 2).

\subsection{Limits to the X-Ray Flux}

Thus far, we have described the X-ray data for each analysis pixel in units of ACIS count rate. Neglecting for the moment pile-up effects, the photon spectral flux (or other related radiation quantity) is proportional to the count rate for an assumed spectral shape. Consequently, any change in count rate corresponds to a proportionate change in photon spectral flux (for an assumed spectral shape). Using the Chandra PIMMS $^{22}$ for the ACIS-S detector and an absorption column $N_{\mathrm{H}}=3.1 \times 10^{21} \mathrm{~cm}^{-2}$, we determine (ignoring pile-up) this

\footnotetext{
22 http://asc.harvard.edu/toolkit/pimms.jsp
}

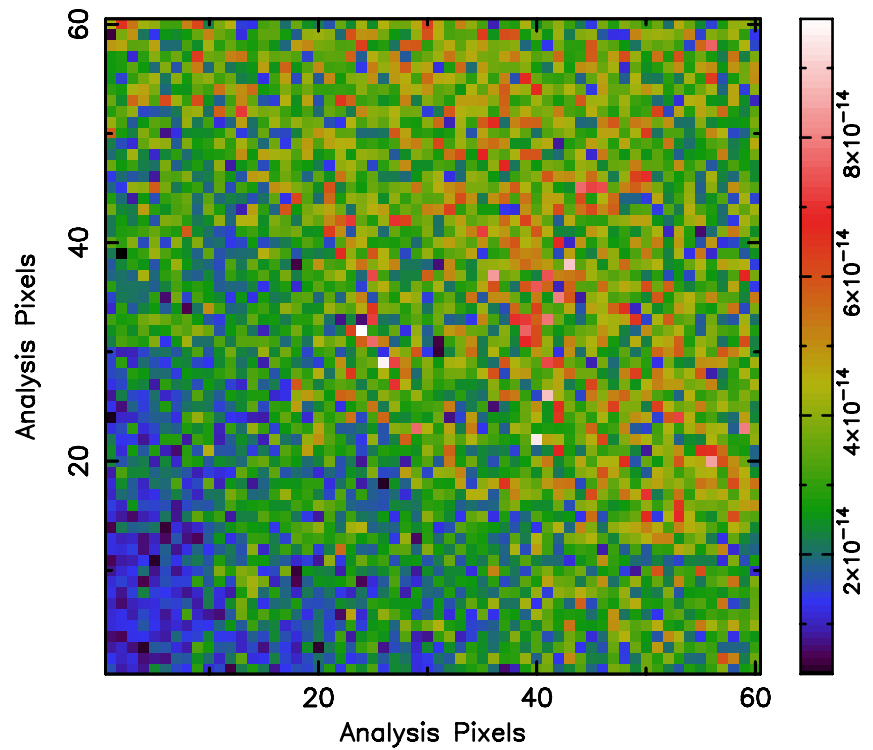

Figure 5. Image of the energy spectral flux $F_{E}\left(E_{x}\right)$, in erg $\left(\mathrm{cm}^{2} \mathrm{~s} \mathrm{keV}\right)^{-1}$ at $E_{x}=1 \mathrm{keV}$, based upon the sample standard deviation $\left(s_{i}\right)$ of the counting rate and assuming $\Gamma_{x}=1$. Note that the indicative energy flux $F(E) \equiv E F_{E}(E)$ in erg $\left(\mathrm{cm}^{2} \mathrm{~s}\right)^{-1}$ happens to have the same numerical value as $F_{E}(E)$ at $E=$ $1 \mathrm{keV}$. North is up and the pulsar is at $(30,30)$ in the displayed analysis-pixel coordinates.

constant of proportionality for an X-ray power-law photon in$\operatorname{dex} \Gamma_{x}=(2 / 3), 1$, and 2: At $E_{x}=1 \mathrm{keV}, N_{E}\left(E_{x}\right) / r=0.99$, 1.26 , and $2.46 \times 10^{-3}$ ph $\left(\mathrm{cm}^{2} \mathrm{~s} \mathrm{keV}\right)^{-1}$ per ct s${ }^{-1}$, respectively. Correcting for pile-up has little effect in low-count-rate regions, but would raise these flux upper limits by $\approx 10 \%$ or so for high-count-rate regions.

Table 2 calculates the photon spectral flux $N_{E}\left(E_{x}\right)$, the energy spectral flux $F_{E}\left(E_{x}\right)$, and the indicative (isotropic) luminosity $E L_{E}\left(E_{x}\right)=4 \pi D^{2} E F_{E}\left(E_{x}\right)$ at $D=2 \mathrm{kpc}$, corresponding to the sample standard deviation and $99 \%$-confidence upper limit for the count-rate variation in the analysis pixel with the most significant $\mathrm{X}$-ray variation. Figure 5 displays an image of the energy spectral flux $F_{E}\left(E_{x}\right)$ at $E_{x}=1 \mathrm{keV}$ for $\Gamma_{x}=1$, based 

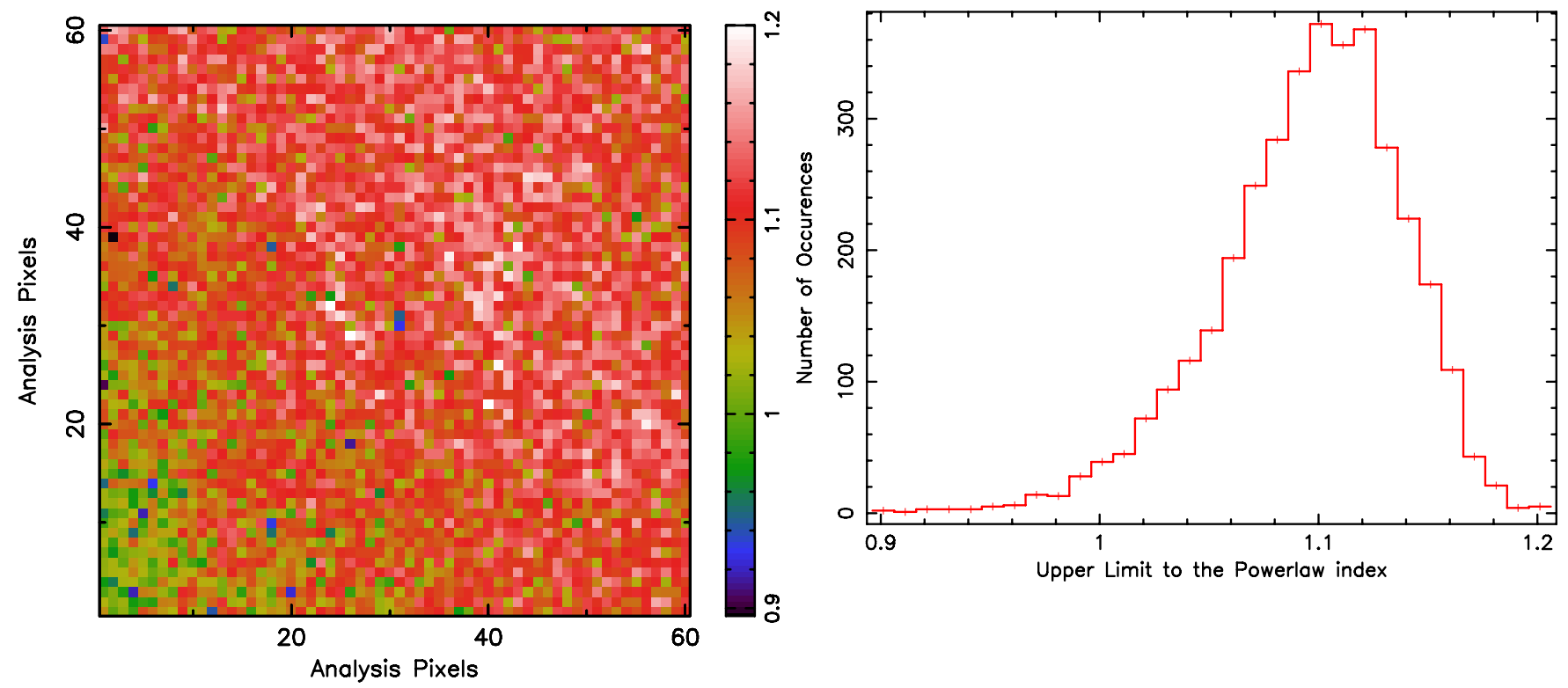

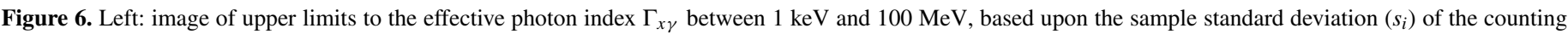

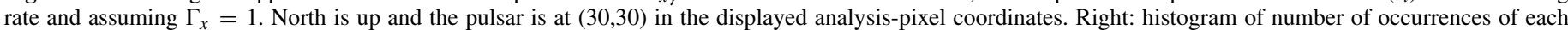
value in the image to the left.

upon the sample standard deviation $s_{i}$ of the count rate in each analysis pixel.

\subsection{Constraints on the $X$-Ray to $\gamma$-Ray Spectral Index}

We now compare the X-ray data with the $\gamma$-ray data to quantify the implications of our lack of detection of time variations in the $\mathrm{X}$-ray data. Our approach compares a variability measure for the X-ray (1 keV) photon spectral flux $\Delta N_{E}\left(E_{x}\right)$ in each analysis pixel with the analogous variability measure for the $\gamma$-ray $(100 \mathrm{MeV})$ photon spectral flux $\Delta N_{E}\left(E_{\gamma}\right)$. In particular, we calculate the sample standard deviation of the $\gamma$-ray spectral flux at $100 \mathrm{MeV}$, using power-law fits to the five Fermi-LAT measurements that were simultaneous with the five Chandra observations. Table 1 lists the five Chandra ObsIDs, their dates, along with the $\gamma$-ray photon index $\Gamma_{\gamma}$, integrated photon flux $N(>100 \mathrm{MeV})$, and photon spectral flux $N_{E}(100 \mathrm{MeV})$. For the five Fermi-LAT observations, the mean and sample standard deviation of the photon spectral flux at $100 \mathrm{MeV}$ are $1.21 \times 10^{-10}$ and $5.77 \times 10^{-11} \mathrm{ph}\left(\mathrm{cm}^{2} \mathrm{~s} \mathrm{keV}\right)^{-1}$, respectively.

Based upon the sample standard deviation $\left(s_{i}\right)$ of photon spectral flux at $E_{x}=1 \mathrm{keV}$ for each X-ray analysis pixel and the measured standard deviation $(5.77 \times$ $\left.10^{-11} \mathrm{ph}\left(\mathrm{cm}^{2} \mathrm{~s} \mathrm{keV}\right)^{-1}\right)$ at $E_{\gamma}=100 \mathrm{MeV}$, we constrain the effective X-ray to $\gamma$-ray photon index of the flaring component: $\Gamma_{x \gamma} \equiv-\log \left[\Delta N_{E}\left(E_{\gamma}\right) / \Delta N_{E}\left(E_{x}\right)\right] / \log \left[E_{\gamma} / E_{x}\right]$. Figure 6 shows the image and corresponding histogram of the distribution of upper limits to $\Gamma_{x \gamma}$ based upon the sample standard deviation of the X-ray measurements and assuming $\Gamma_{x}=1$.

In that the $\gamma$-ray variations are statistically significant and the X-ray variations are not, we compute $99 \%$-confidence upper limits to $\Gamma_{x \gamma}$ (Table 2, last row). Note that the upper limits to $\Gamma_{x \gamma}$ are marginally consistent with the low-energy extrapolation of the $\gamma$-ray spectrum $\left(\Gamma_{\gamma}=1.27 \pm 0.12\right)$ of the flaring component (Buehler et al. 2012).

\subsection{Variability within an $X$-Ray Image}

In Sections 2.1 and 2.2, the search for variability focused on sensitivity to flux changes amongst the five pointings with a minimum cadence of 0.6 days. Here, we search for variability on shorter timescales-namely within each pointing. As described in Section 2, the ACIS S3 CCD was read at most roughly 6000 times in each pointing due to telemetry saturation (dead time). In this study, rather than using the $2 \times 2$ ACIS analysis pixel, we employed a circular search bin with a radius of 1 ACIS-pixel (0'49) on a grid with 0.1 ACIS pixel spacing. Note that this oversampling implies that the results of the test in adjacent pixels are not statistically independent. (We also analyzed these data using the statistically independent analysis pixels of Section 2.1 with similar results as below.)

Using the frame number of each detected photon, we derive the empirical cumulative distribution function (ECDF) of the frames with a photon arriving in the analysis pixels of the CCD. This ECDF is then compared with the corresponding ECDF of the exposure given by the sequence of frames actually read. Finally, we compare the two ECDFs using a Kolmogorov-Smirnov test, resulting in a probability estimate $Q$ that the two ECDFs are derived from the same parent distribution. A low value of $Q$ would indicate possible variability. The results of the test for the five pointings were very similar. The smallest value, $Q_{\min }=6.7 \times 10^{-7}$, was obtained in observation 13151. Note that selecting this point represents a tuning bias, as the noise in neighboring points is highly correlated due to the oversampling. The probability of finding at least one pixel with $Q_{\text {min }}$ considering that there are $120 \times 120 /\left(\pi \times 1^{2}\right) \times 5$ statistically independent trials is 0.015 , which we regard as a lower limit due to the tuning bias. A 0.015 probability is tantalizing but not compellingly significant: hence, we do not claim detection of short-timescale variability. The fact that the location of the point with minimum $Q$ is very close to the pulsar, a region in which pileup plays a strong role in blotting out the image, bolsters our somewhat conservative conclusion.

\section{NEAR-INFRARED IMAGE OF THE INNER KNOT}

The extreme saturation of the pulsar in the X-ray images means that we cannot easily study the central $2^{\prime \prime}$ in X-rays. However, this region does contain a nebular structure of particular interest: the "inner knot" whose peak is 0'.65 southeast 


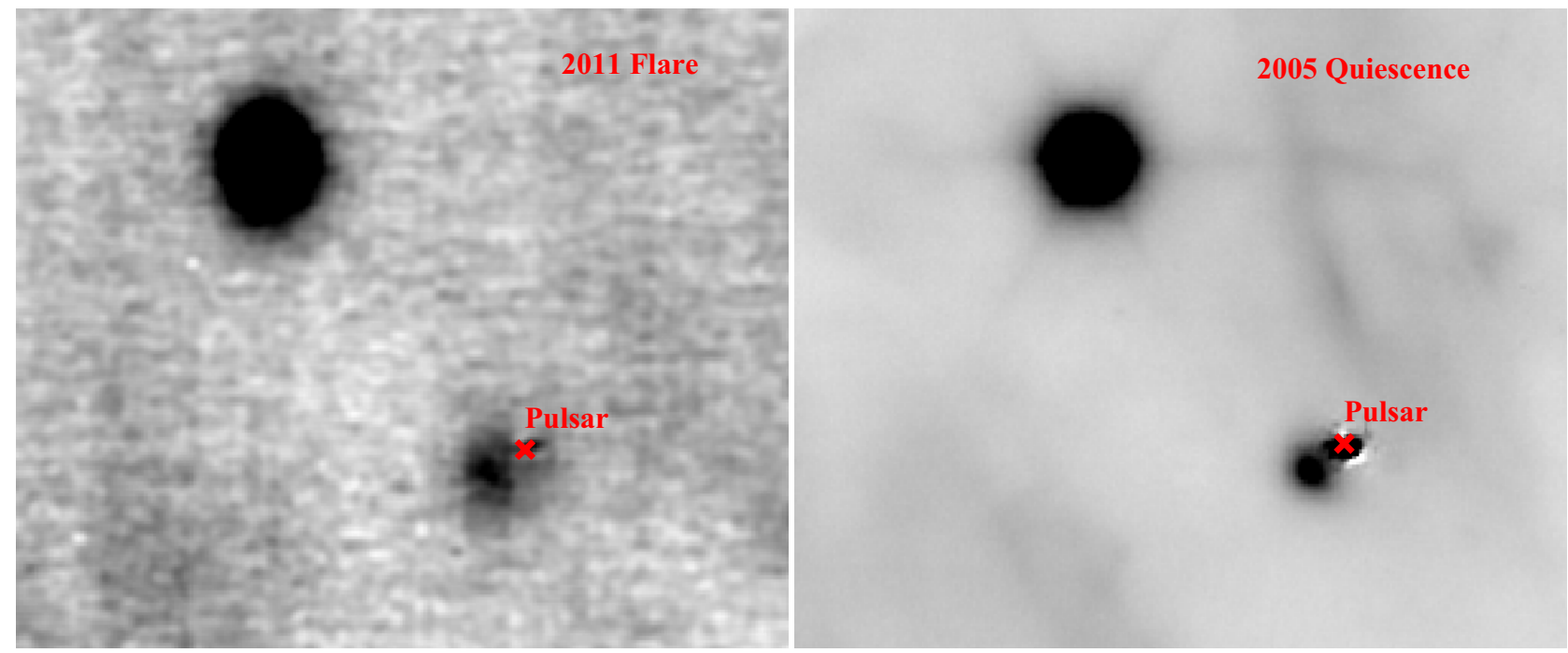

Figure 7. Keck $K^{\prime}$ observations of the Crab, after subtraction of a field-star image from the pulsar position (marked with $\mathrm{x}$ ). Left: (MJD-50814) $=4853.250$ without adaptive optics $(\mathrm{AO})$. Right: (MJD-50814) $=2870$, with laser guide star AO. Residuals from imperfect subtraction of the point-spread function are visible at the pulsar position; the "inner knot" is the extended structure to the southeast.

of the pulsar at position angle $118^{\circ}$ east from north (Hester 2008). This structure, an oval shape extending $\approx 0$ ' 75 , is well measured in $H S T$ and ground-based near-IR images. Given its relatively red spectrum (energy spectral index $\alpha_{\nu}=-1.3 \pm 0.1$ versus $\alpha_{v}=0.27 \pm 0.03$ for the pulsar; Sandberg \& Sollerman 2009), it is one of the near-IR brightest structures in the Nebula. Sandberg \& Sollerman (2009) note that the knot varies by a factor of two; we confirm typical variability of $20 \%-30 \%$ in archival HST images. Komissarov \& Lyutikov (2011) have proposed that this structure represents radiation from an oblique termination shock in the pulsar wind nebula. In this picture, the Earth line-of-sight is tangent to the flow at the inner knot position, and thus the intensity experiences substantial Doppler boosting for synchrotron emission in the mildly relativistic postshock flow. Indeed, in relativistic MHD simulations they find that this bright spot is highly variable and can dominate the $\gamma$-ray synchrotron emission. Alternatively, the knot could be a time varying standing shock in the polar jet flow itself, a flow known to be highly variable from HST imaging (Hester et al. 1995, 2002; Hester 2008).

It is thus of interest to check the status of the knot during the 2011 April $\gamma$-ray flare. Unlike the sequence of multiwavelength observations performed after the 2010 September flare, it was impossible to trigger an allocated HST Target of Opportunity observation owing to solar constraints in April. We were able to obtain a Keck Near Infrared Camera (NIRC2) $K^{\prime}$ exposure (Figure 7, left image) on MJD 55667.250, almost precisely at the peak of the $\gamma$-ray flux and $2.5 \mathrm{hr}$ before the ACIS image ObsID 13152 (Figure 1). Unfortunately, the observations occurred during twilight and only one $20 \times 4 \mathrm{~s}$ integration without dithering was obtained. Under these conditions the adaptive optics (AO) loop did not close, leaving an undithered image with native 0'.46 FWHM seeing. This frame was dark subtracted and an approximate background was removed using an immediately subsequent image. Despite the modest image quality, the inner knot was well detected. After subtracting the pulsar with a scaled image of the comparably bright companion star $4^{\prime \prime}$ northeast, we measured the knot flux and position. We find a magnitude $K^{\prime}=15.60 \pm 0.03$ and an offset $0^{\prime \prime} 64 \pm 0.0^{\prime \prime} 04$ from the pulsar. For comparison, we measured a high-quality NIRC2 $K^{\prime}$ image (Figure 7, right image) obtained 2005 November 10. Here the

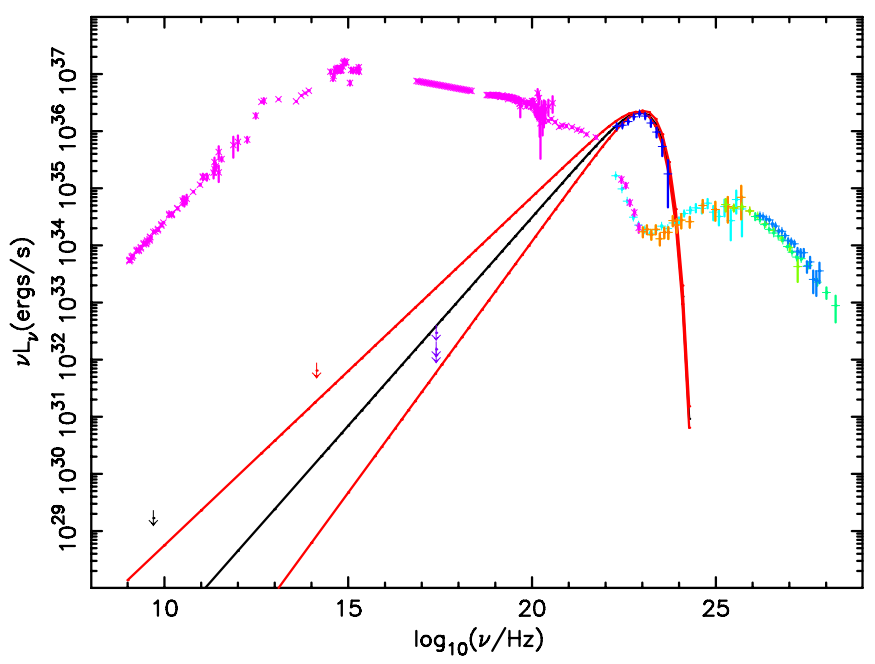

Figure 8. Spectral energy distribution (SED) of archival data (purple, orange, cyan, light green, turquoise, light blue) compiled by Meyer et al. (2010). Power is scaled from flux assuming isotropic emission at $2 \mathrm{kpc}$. The Fermi-LAT data for the 2011 April flare component appear in dark blue (Buehler et al. 2012). The solid black and red curves are fits to the flare spectrum with a power-law extrapolation to lower energies of photon index $\Gamma=1.27 \pm 0.12$ (spectrum 7 in Buehler et al. 2012). The three downward blue arrows at $\log _{10} v=17.4$ mark $99 \%$-confidence upper limits to a variable X-ray component, in increasing $E L_{E}=\nu L_{v}$ for $\Gamma_{x}=(2 / 3), 1$, and 2, respectively. (NB: values for $E L_{E}$ from Table 2 are multiplied by $2 \sqrt{2}$ to scale from a standard deviation to a peak-to-valley, for comparison with the plotted SED of the $\gamma$-ray flare.) The red downward arrow at $\log _{10} v=14.1$ indicates an upper limit to infrared variability of the inner knot, determined from the difference between the two Keck images. Finally, the black downward arrow at $\log _{10} v=9.7$ gives an upper limit to $5 \mathrm{GHz}$ radio variability, based upon the April 15 VLA measurement.

knot is $K^{\prime}=15.94 \pm 0.02$ at offset $0.58 \pm 00^{\prime} 02$. We also note that Sandberg \& Sollerman (2009) measured $K_{s}=15.80 \pm 0.03$ on 2003 October 18 . We conclude that the knot was in a relatively bright state during the flare $(\approx 35 \%$ brighter than in 2005), but well within the normal range of flux (and position) variation. Thus, there is no dramatic change in the inner knot in the nearIR band. We use the amplitude of the measured variation as an upper limit to any variation in the inner knot associated with the $\gamma$-ray flare (Figure 8). 
Table 3

VLA Point-source Detections and Limits in the Crab Field

\begin{tabular}{lcccc}
\hline \hline $\begin{array}{l}\text { Epoch } \\
\text { (in 2011) }\end{array}$ & Date $^{\mathrm{a}}$ & Array & $\begin{array}{c}\text { Frequency } \\
(\mathrm{GHz})\end{array}$ & $\begin{array}{c}\text { Detection or Limit } \\
(\mathrm{mJy})\end{array}$ \\
\hline Apr 15 & 4852.980 & B & 4.959 & $<7.1$ \\
Apr 19 & 4856.006 & B & 4.195 & $<5.7$ \\
Apr 19 & 4856.006 & B & 7.795 & $<1.7$ \\
Apr 21 & 4858.011 & B & 4.195 & $<4.3$ \\
Apr 21 & 4858.011 & B & 7.795 & $<1.0$ \\
Apr 22 & 4859.919 & B & 4.910 & $1.78 \pm 0.086$ \\
Apr 22 & 4859.919 & B & 8.566 & $0.52 \pm 0.094$ \\
Apr 30 & 4867.901 & B & 4.195 & $<7.9$ \\
Apr 30 & 4867.901 & B & $7.762^{b}$ & $5.55 \pm 0.86$ \\
Apr 30 & 4867.901 & B & $7.827^{b}$ & $4.59 \pm 0.96$ \\
May 12 & 4879.985 & BnA & 4.195 & $<2.1$ \\
May 12 & 4879.985 & BnA & 7.795 & $<0.8$ \\
May 12 & 4879.985 & BnA & 22.46 & $<0.4$ \\
May 28 & 4895.925 & BnA & 4.195 & $<2.4$ \\
May 28 & 4895.925 & BnA & 7.795 & $<1.2$ \\
Jul 10 & 4938.791 & A & 4.195 & $<0.7$ \\
Jul 10 & 4938.791 & A & 7.795 & $<1.1$ \\
Jul 10 & 4938.791 & A & 22.46 & $<1.7$ \\
\hline
\end{tabular}

Notes.

a Days after MJD 50814 to the middle of the on-target exposures.

b For the 7.8 GHz detection on April 30, the data were further split into two sub-bands.

c Limits list $3 \sigma$ uncertainties. Detections list $1 \sigma$ uncertainties; corresponding point-source limits during these two epochs should take three times the $1 \sigma$ values.

\section{RADIO OBSERVATIONS}

On 2011 April 14, we triggered a prompt radio follow-up program with the VLA. The VLA observations occurred in eight epochs starting April 15 and ending July 10. These observations detected the pulsar at two epochs, but found no other point sources in the field. Observations were predominantly in the range 4-8 GHz, with additional observations at $1.4 \mathrm{GHz}$ (not reported; see below) and at $22 \mathrm{GHz}$ (for some later epochs). Unless otherwise noted, all observations used two sub-bands, each with a $128 \mathrm{MHz}$ bandwidth. In each run, observations of the target were bracketed with scans of a phase calibrator (J0559+2353, except where noted) and a flux calibrator (3C 147). The fields of view are limited to the primary beam response of the antennas, with full width at half-power of $9^{\prime} / \nu_{5}$ with $\nu_{5} \equiv v /(5 \mathrm{GHz})$. Table 3 provides a summary of the observational parameters and results.

Our initial observations were obtained through a Fermi guest-investigator cycle-3 program (S3184) approved for four $1 \mathrm{hr}$ runs in the $L$-band and $C$-band $(\approx 1.4$ and $5 \mathrm{GHz}$, respectively). The VLA was in its B-array configuration during these observations, resulting in images with angular resolution $\approx 1^{\prime \prime} / \nu_{5}$. We found that the $L$-band data for the Crab were highly confused due to the brightness and complexity of the steepspectrum nebular emission in the first (April 15) and second (April 19) epochs. Consequently, we modified our strategy for subsequent observations. After the first epoch, we split the $C$-band observations into two widely spaced side-bands centered at 4.2 and $7.8 \mathrm{GHz}$, aiming better to constrain the spectrum of any detected source. We also began scheduling observations only at frequencies greater than $4 \mathrm{GHz}$ after the second epoch. In these B-array data, our point-source limits at the lower frequency are $\approx 3-4 \times$ larger than at the higher frequency, again due to the Crab Nebula's steep-spectrum radio emission.
After non-detection of any significant radio point-source emission down to $\approx 1-7 \mathrm{mJy}(3 \sigma)$ sensitivities in the initial three VLA observations 1-7 days after the $\gamma$-ray peak (Hays et al. 2011), we purposely delayed the fourth observation until 9 days after the previous observation to probe longer timescales. Only in this last observation (April 30) did we obtain a significant point-source detection, which was coincident with the Crab pulsar position, but only in the upper side-band centered at 7.8 GHz. The detection is a factor of five greater than the $3 \sigma$ limit from nine days prior. The source was not detected at the lower frequency side-band $(4.2 \mathrm{GHz})$ with a limit indicating a source with a flat radio spectrum.

Following the point-source detection on April 30, we became aware of VLA TEST observations of the Crab obtained on April 22 (program TDEM0007; PI: D. Frail). These data were obtained with wide bandwidth $(16 \times 128 \mathrm{MHz}$ wide sidebands $)$, so were more sensitive than those from our observing runs. The flux densities were scaled to 3C 147; J0534+1927 was utilized for phase calibration. The flat-spectrum radio source coincident with the Crab pulsar detected in our Apr-30 observation was confirmed in the Apr-22 data in two bands, but with a much lower $(10 \times)$ flux. Also, the source spectrum was rather steep, with an energy spectral index $\alpha=2.21 \pm 0.34\left(S_{v} \propto v^{-\alpha}\right)$ between $5 \mathrm{GHz}$ and $8.6 \mathrm{GHz}$.

Following the radio detections of the pulsar, we requested further VLA monitoring of the Crab through Director's Discretionary Time (program 11A-268 = AC1052). In addition to the $C$-band observations, we obtained exposures in the $K$ band (centered at 22.396 and $22.254 \mathrm{GHz}$ ) aiming to constrain further the spectrum of any detected radio source. Through this program, we obtained $2 \mathrm{hr}$ runs on May 12-13 (while the VLA was in its hybrid BnA array) and on July 10-11 (in A array), and an additional $1 \mathrm{hr}$ run on May 28, using one of the early (April 19, from program S3184) frequency setups. An angular resolution of $\approx\left(0,3 / v_{5}\right)$ is typically achieved in A-array VLA observations. With the higher resolution, we obtained systematically $4 \times$ lower flux limits than in the lower resolution B-array data, presumably due to lesser contribution from the extended nebular emission. In none of these later epoch follow-up observations, did we detect a point source, to typical limits of 1-2 mJy at each of the three frequencies (see Table 3).

\subsection{Discussion of the Radio Data}

Previously, it was argued that the $\gamma$-ray flaring possibly originates in a knot 5.7 east of the pulsar (Tavani et al. 2011). Indeed, this knot is the site of the most significant X-ray variability we observe (Section 2.2) during the 2011 April flaring episode. Variable radio emission was detected around the time of the previous Crab $\gamma$-ray flaring episode (Lobanov et al. 2011) with fainter flux densities than achieved in our VLA observations. However, we found no significant radio point-source counterpart to this knot in any of our eight epoch VLA observations following the 2011 April $\gamma$-ray flare. Rather, we detected a variable continuum radio source with the VLA, coincident within $0^{\prime} .2$ of the Crab pulsar position in two of eight epochs. However, the flux level and cadence of the radio detections is consistent with previous observations by Moffett $\&$ Hankins (1996), who detected the pulsar $20 \%-40 \%$ of the times they observed. Moreover, the dates of the radio point-source detections coincident with the pulsar do not coincide with any feature in the $\gamma$-ray light curve (Figure 1), having occurred $8-16$ days after the brightest $\gamma$-ray peak. Consequently, our 
VLA follow-up observations provide no conclusive evidence for the site of the $\gamma$-ray flares.

\section{DISCUSSION}

Here we discuss possible explanations for the absence in non$\gamma$-ray bands of variability that is obviously correlated with the $\gamma$-ray flare. In addition we present a conceptual model for the production of the $\gamma$-ray flares.

\section{1. $\gamma$-Ray Emission}

As was recognized immediately, the SED of $\gamma$-ray flares peak near a characteristic energy about five times the energy $\alpha^{-1} m_{e} c^{2} \approx 70 \mathrm{MeV}$, which is identified with radiationreaction-limited synchrotron (magneto-bremsstrahlung) emission (e.g., Landau \& Lifshitz 1959). Subjected to comparable parallel and perpendicular electromagnetic acceleration, an electron radiates at this energy, independent of the strength of the acceleration. An electron emitting synchrotron radiation in a magnetostatic field with peak emission at several $100 \mathrm{MeV}$ would cool in turning through $\approx 0.2 \mathrm{rad}$ and would thus require a parallel electric field $E \approx 5 c B$ to compensate the radiative loss.

The Crab pulsar releases energy in an essentially electromagnetic form. Poynting flux flows radially outward from the pulsar through the light cylinder at $c P /(2 \pi) \approx 1500 \mathrm{~km}$ and into an outflowing wind, where at least some of the electromagneticenergy flux may transform into a plasma-energy flux. How, where, and to what extent this happens has long been a matter of debate (e.g., Arons 2010; Kirk et al. 2009). Furthermore, the electromagnetic component has a DC toroidal part with an associated quadrupolar current distribution, and an AC, "striped" part containing current sheets separated by $(1 / 2) c P \approx 5000 \mathrm{~km}$. The transformation from electromagnetic to plasma energy might be non-dissipative-through the action of a Lorentz force (e.g., Bogovalov 1997; Bogovalov 2001)—or dissipative-through particle heating and acceleration (e.g., Coroniti 1990; Lyubarsky \& Kirk 2001; Sironi \& Spitkovsky 2011). However, it must occur somewhere as magnetic flux would otherwise accumulate in the nebula, ultimately reacting back on the pulsar. Some of this transformation from electromagnetic to plasma energy may occur at a shock (Pétri \& Lyubarsky 2007; Sironi \& Spitkovsky 2011) with radius $\approx 10^{17} \mathrm{~cm}$, where the wind momentum flux balances the ambient nebular pressure (Rees \& Gunn 1974; Kennel \& Coroniti 1984). It has also been proposed that the toroidal field loops contract to form an axial pinch (identified with the X-ray jet) and reconnect at an equatorial current sheet (the torus) (Komissarov \& Lyubarsky 2003; Del Zanna et al. 2004; Camus et al. 2009).

In many respects the pulsar is a current generator. The supersonic wind contains outflowing fluxes of electrons and positrons. (Any ions that are present behave like positrons of similar rigidity but do not radiate.) The difference in their fluxes determine the current density. This current may concentrate into sheets and filaments, where strong dissipation can occur-as happens in heliospheric and laboratory plasmas (Gosling et al. 2005; Sui \& Holman 2003; Sergeev et al. 1993). In particular, the inner wind, the shock, the jet, and the torus are all natural sites of rapid dissipation and $\gamma$-ray emission. If we consider this dissipation more generally under electromagnetic conditions, a current $I$ may be associated with a potential difference $V \approx I Z_{0}$ where $Z_{0}=\mu_{0} c=377 \Omega$ is the impedance of free space and we drop model-dependent constants of order unity. (This result can be anticipated on the basis of dimensional analysis or exhibited in particular simple cases.) The maximum energy to which an electron or positron can be accelerated is $\gamma_{\max } m_{e} c^{2} \approx \mathrm{eV} \approx e I Z_{0}$ and the expected power is then $L \approx I V \approx I^{2} Z_{0}$. On this basis, a spectrum of currents extending up to $\approx 30$ TA should suffice to account for the $\gamma$-ray variations.

However, currents do not automatically dissipate as just described. Large electric fields are normally discharged in a few plasma periods. The best way to create them here is transiently over a few Larmor periods and radii. This, in turn, requires local charge separation of the plasma in the emission site. To be more precise, the density $n=n_{-}+n_{+}$of electrons plus positrons/ ions will combine to create a local current density $j \approx n e c$. For example, in the case of a pinch, the gradient, polarization and curvature drifts automatically produce the axial current. However, a supporting local electric field of strength $E \approx c B$ also requires that $\left|n_{-}-n_{+}\right| \approx n$.

Put another way, the charge and current are mostly in an emission site that is a few Larmor radii in size and survives for a few Larmor periods of the $\gamma$-ray emitting particles. When this happens, the "Ohmic" dissipation is radiative, not collisional as is normally the case. For this to occur, the particles must be sufficiently energetic to radiate efficiently. This requires that most of the particles are concentrated in an emission site that is as small as $\approx \gamma_{\max }^{3} r_{e} \approx\left(e I Z_{0} / m_{e} c^{2}\right)^{3} r_{e} \approx 10^{16} \mathrm{~cm}$. The key point is that there should be extensive and sustained radiationreaction-limited emission at the peak $\gamma$-ray-flare energy, even though the particle energy and magnetic field might be changing. Note that when this condition is unsatisfied, efficient particle acceleration to lower energy should still result: Most of the magnetic dissipation and particle acceleration in the nebula might occur in this fashion. Detailed modeling is necessary to determine whether or not such a scheme can reproduce the powerful, narrowband $\gamma$-ray variation that is observed (Uzdensky et al. 2011; Cerutti et al. 2012a, 2012b; Lyutikov et al. 2012; Bykov et al. 2012; Sturrock \& Aschwanden 2012; R. Blandford \& Y. Yuan, in preparation) and to see if unstable magnetized plasmas, carrying large currents, evolve to satisfy these conditions.

\subsection{Associated Emission}

Whether we interpret the $\gamma$-rays as coming from radiationreaction-limited synchrotron emission or simply extrapolate the observed $\gamma$-ray spectra to lower energy, it should not be surprising that direct, associated emission has not yet been observed in the X-ray, optical, or radio bands: In these bands the contrast with the steady emission is too small to be easily noticed. However, the indirect effects could be larger and detectable. For example, the large 2011-April flare produced a radiant energy of $6 \times 10^{40} \mathrm{erg}$ if isotropic, equivalent to the energy contained within a region of size $\approx 2 \times 10^{16}$ $\mathrm{cm}$ subtending an angle $\approx 0$ '.3. It seems unlikely that the dynamical aftermath of a major flare would not alter the ambient emission-either through compression or rarefaction that would cause the magnetic field strength and the electron distribution function to change significantly. The associated surface brightness change should be several percent, assuming a total emission region of size $\approx 0$ ' 3 , consistent with our upper limits. Even if future observations fail to exhibit associated emission, they may still rule out specific detailed mechanisms in local sites.

Understanding the emission mechanism could have a significance beyond pulsar wind nebulae. In particular, it could provide 
a clue to the surprisingly rapidly variable emission seen in relativistic jets in the radio, optical, $\mathrm{X}$-ray, and $\mathrm{TeV}$ bands. If so, the Crab Nebula would once again be the source of fresh and important astrophysical insight.

\section{SUMMARY}

Using the Chandra, Keck, and VLA Observatories, we acquired X-ray, near-IR, and radio images of the Crab Nebula, contemporaneous with the 2011 April $\gamma$-ray flare. We searched for variability in the X-ray data over two timescale ranges: First we tested for pointing-to-pointing variations amongst the five pointings, each with an effective exposure time $\approx 1200 \mathrm{~s}$ and a minimum separation of 0.6 days. Second, we tested for variations within each of the five observations. In neither case did we detect statistically significant X-ray variations; thus we can set only upper limits to any X-ray variations associated with the $\gamma$-ray flare. As the Chandra ACIS images suffer severe pileup near the Crab pulsar, our search for variability in the X-ray images was not sensitive to variations within the central $\approx 1^{\prime \prime} .5$ or so.

Comparing the upper limits to X-ray variations with the Fermi-LAT-measured $\gamma$-ray variations, we set upper limits at $99 \%$ confidence to the effective X-ray- $\gamma$-ray photon powerlaw index $\Gamma_{x \gamma} \leqslant 1.20$ to $\leqslant 1.27$, dependent upon assumptions about the X-ray index $\Gamma_{x}$. As Fermi-LAT measures a $\gamma$-ray index $\Gamma_{\gamma}=1.27 \pm 0.12$ for the flaring component, it is statistically possible that the flaring component's spectrum extends as a simple power-law from $\gamma$-rays to X-rays. Further, we note that our upper limit to $\Gamma_{x \gamma}$ is consistent with transparent synchrotron emission, whose photon index must be $>(2 / 3)$.

Comparison of two Keck near-IR observations found that the inner knot $(\approx 0$ '. 65 from the pulsar) was somewhat brighter than average during the $\gamma$-ray flare, but well within the normal range of brightness fluctuations typically observed. We used the measured $(\approx 35 \%)$ change in the near-IR flux from this knot as an upper limit to near-IR variations associated with the $\gamma$-ray flare. We also performed a number of VLA observations searching for a point source appearing either at an unusual location and/or contemporaneous with the $\gamma$-ray flare. Other than the pulsar itself, no such source was detected.

Figure 8 shows the SED of the Crab Nebula over the observed electromagnetic spectrum. The plot also shows the SED of the 2011 April $\gamma$-ray flare and the various limits determined here on variable radio, near-infrared, and X-ray emission possibly associated with the $\gamma$-ray flare.

Finally, we reviewed and discussed potential implications of $\gamma$-ray flares and theoretical issues to be addressed. We concluded that, apart from lower-energy emission directly associated with the $\gamma$-ray flare itself, the dynamical aftermath of a major flare could alter the ambient emission-e.g., through compression of the magnetic field. The associated surface brightness change would likely be only several percent, assuming a total emission region of size $\approx 0$ '!3, consistent with our upper limits.

Although no "smoking gun" has been identified, one should be encouraged that we have identified a number of regions in the X-ray images that are possible candidates. We have also established further Target of Opportunity observations with Chandra and HST that will be triggered at the onset of the next $\gamma$-ray flare. The X-ray observations will also probe the region very close to the pulsar using the Chandra High-Resolution Camera (HRC).
The work of M.C.W., S.L.O., and A.F.T. is supported by the Chandra Program. The Chandra data was obtained in response to a pre-approved target of opportunity request granted under Chandra Director's Discretionary Time. The Fermi LAT Collaboration acknowledges generous ongoing support from a number of agencies and institutes that have supported both the development and the operation of the LAT as well as scientific data analysis. These include the National Aeronautics and Space Administration and the Department of Energy in the United States, the Commissariat à l'Energie Atomique and the Centre National de la Recherche Scientifique/Institut National de Physique Nucléaire et de Physique des Particules in France, the Agenzia Spaziale Italiana and the Istituto Nazionale di Fisica Nucleare in Italy, the Ministry of Education, Culture, Sports, Science and Technology (MEXT), High Energy Accelerator Research Organization (KEK) and Japan Aerospace Exploration Agency (JAXA) in Japan, and the K. A. Wallenberg Foundation, the Swedish Research Council and the Swedish National Space Board in Sweden. Additional support for science analysis during the operations phase is gratefully acknowledged from the Istituto Nazionale di Astrofisica in Italy and the Centre National d'Études Spatiales in France. C.C.C., G.B.T., and J.D.L. thank Tim Hankins for useful discussions, and the NRAO scheduling committee for alerting us to the VLA TEST data and for their prompt consideration of our Director's Discretionary Time observations. C.C.C., G.B.T., and J.B.L. were supported in part by NASA through a Fermi cycle-3 guest investigator grant. In addition, work by C.C.C. at NRL is supported in part by NASA DPR S-15633-Y. Our analyses utilized software tools provided by the Chandra X-ray Center (CXC) in the application package CIAO and from the High-Energy Astrophysics Science Archive Research Center (HEASARC, operated by the NASA Goddard Space Flight Center, Greenbelt, MD, and by the Smithsonian Astrophysical Observatory, Cambridge, MA).

Facilities: CXO, Fermi, VLA

\section{REFERENCES}

Abdo, A. A., Ackermann, M., Ajello, M., et al. 2010, ApJ, 708, 1254 Abdo, A. A., Ackermann, M., Ajello, M., et al. 2011, Sci, 331, 739 Aharonian, F., Akhperjanian, A., Beilicke, M., et al. 2004, ApJ, 614, 897 Albert, J., Aliu, E., Anderhub, H., et al. 2008, ApJ, 674, 1037 Arons, J. 2010, in COSPAR Meeting, Vol. 38, 38th COSPAR Scientific Assembly, held 8-15 July 2010 in Bremen, Germany, 2

Atoyan, A. M., \& Aharonian, F. A. 1996, MNRAS, 278, 525

Bogovalov, S. V. 1997, A\&A, 327, 662

Bogovalov, S. V. 2001, A\&A, 367, 159

Bucciantini, N., Arons, J., \& Amato, E. 2011, MNRAS, 410, 381

Buehler, R., Scargle, J. D., Blandford, R. D., et al. 2012, ApJ, 749, 26

Bykov, A. M., Pavlov, G. G., Artemyev, A. V., \& Uvarov, Y. A. 2012, MNRAS, 421, L67

Camus, N. F., Komissarov, S. S., Bucciantini, N., \& Hughes, P. A. 2009, MNRAS, 400, 1241

Caraveo, P., de Luca, A., Mignani, R., et al. 2010, ATel, 2903

Caraveo, P. A., \& Mignani, R. 1999, A\&A, 344, 367

Cerutti, B., Uzdensky, D. A., \& Begelman, M. C. 2012a, ApJ, 746, 148

Cerutti, B., Werner, G. R., Uzdensky, D. A., \& Begelman, M. C. 2012b, ApJL, 754, L33

Coroniti, F. V. 1990, ApJ, 349, 538

Cusumano, G., La Parola, V., Romano, P., Burrows, D. N., \& Gelbord, J. M. 2011, ATel, 3279

Del Zanna, L., Amato, E., \& Bucciantini, N. 2004, A\&A, 421, 1063

Evangelista, Y., Campana, R., Capalbi, M., et al. 2010, ATel, 2866

Ferrigno, C., Tennant, A., Horns, D., et al. 2010, ATel, 2994

Gosling, J. T., Skoug, R. M., McComas, D. J., \& Smith, C. W. 2005, JGRA, 110,1107

Hays, E., Buehler, R., \& D’Ammand, F. 2011, ATel, 3284

Hester, J. J. 2008, ARA\&A, 46, 127

Hester, J. J., Mori, K., Burros, D., et al. 2002, ApJL, 577, L49 
Hester, J. J., Scowen, P. A., Sankrit, R., et al. 1995, ApJ, 448, 240

Horns, D., Tennant, A., Ferrigno, C., et al. 2010, ATel, 3058

Kanbach, G., Kruehler, T., Steiakaki, A., \& Mignani, R. 2010, ATel, 2867

Kaplan, D. L., Chatterjee, S., Gaensler, B. M., \& Anderson, J. 2008, ApJ, 677, 1201

Kennel, C. F., \& Coroniti, F. V. 1984, ApJ, 283, 694

Kirk, J. G., Lyubarsky, Y., \& Petri, J. 2009, in Neutron Stars and Pulsars, ed. W. Becker (Astrophysics and Space Science Library, Vol. 357; Berlin: Springer), 421

Komissarov, S. S., \& Lyubarsky, Y. E. 2003, MNRAS, 344, L93

Komissarov, S. S., \& Lyutikov, M. 2011, MNRAS, 414, 2017

Landau, L. D., \& Lifshitz, E. M. 1959, The Classical Theory of Fields, Vol. 2 (2nd ed.; Reading, MA: Pergamon Press)

Lobanov, A. P., Horns, D., \& Muxlow, T. W. B. 2011, A\&A, 533, A10

Lyubarsky, Y., \& Kirk, J. G. 2001, ApJ, 547, 437

Lyutikov, M., Balsara, D., \& Matthews, C. 2012, MNRAS, 422, 3118

Meyer, M., Horns, D., \& Zechlin, H.-S. 2010, A\&A, 523, A2

Moffett, D. A., \& Hankins, T. H. 1996, ApJ, 468, 779
Ng, C.-Y., \& Romani, R. W. 2007, ApJ, 660, 1357

Pétri, J., \& Lyubarsky, Y. 2007, A\&A, 473, 683

Rees, M. J., \& Gunn, J. E. 1974, MNRAS, 167, 1

Sandberg, A., \& Sollerman, J. 2009, A\&A, 504, 525

Sergeev, V. A., Mitchell, D. G., Russell, C. T., \& Williams, D. J. 1993, JGR, 98, 17345

Shaposhnikov, N., Jahoda, K., Swank, J., et al. 2010, ATel, 2872

Sironi, L., \& Spitkovsky, A. 2011, ApJ, 741, 39

Striani, E., Piano, G., Tavani, M., et al. 2011a, ATel, 3286

Striani, E., Tavani, M., Piano, G., et al. 2011b, ApJL, 741, L5

Sturrock, P., \& Aschwanden, M. J. 2012, ApJL, 751, L32

Sui, L., \& Holman, G. D. 2003, ApJL, 596, L251

Tavani, M., Bulgarelli, A., Vittorini, V., et al. 2011, Sci, 331, 736

Tennant, A., Blandford, R., Buehler, R., et al. 2011, ATel, 3283

Tennant, A., Caraveo, P., Costa, E., et al. 2010, ATel, 2882

Uzdensky, D. A., Cerutti, B., \& Begelman, M. C. 2011, ApJL, 737, L40

Weisskopf, M. C., Hester, J. J., Tennant, A. F., et al. 2000, ApJL, 536, L81

Wilson-Hodge, C. A., Cherry, M. L., Case, G. L., et al. 2011, ApJL, 727, L40 